\title{
Finite and Uniform Stability of Sphere Packings*
}

\author{
A. Bezdek, ${ }^{1}$ K. Bezdek, ${ }^{2,3}$ and R. Connelly ${ }^{3}$ \\ ${ }^{1}$ The Mathematical Institute, Hungarian Academy of Sciences, \\ Budapest, Hungary \\ and \\ Department of Mathematics, Auburn University, \\ AL, USA \\ bezdean@mail.auburn.edu \\ ${ }^{2}$ Department of Geometry, Eötvös University, \\ Budapest, Hungary \\ kbezdek@ludens.elte.hu \\ ${ }^{3}$ Department of Mathematics, Cornell University, \\ Ithaca, NY 14853, USA \\ \{kbezdek,connelly\}@math.cornell.edu
}

\begin{abstract}
The main purpose of this paper is to discuss how firm or steady certain known ball packing are, thinking of them as structures. This is closely related to the property of being locally maximally dense. Among other things we show that many of the usual best-known candidates, for the most dense packings with congruent spherical balls, have the property of being uniformly stable, i.e., for a sufficiently small $\varepsilon>0$ every finite rearrangement of the balls of this packing, where no ball is moved more than $\varepsilon$, is the identity rearrangement. For example, the lattice packings $D_{d}$ and $A_{d}$ for $d \geq 3$ in $E^{d}$ are all uniformly stable. The methods developed here can work for many other packings as well. We also give a construction to show that the densest cubic lattice ball packing in $E^{d}$ for $d \geq 2$ is not uniformly stable.

A packing of balls is called finitely stable if any finite subfamily of the packing is fixed by its neighbors. If a packing is uniformly stable, then it is finitely stable. On the other hand, the cubic lattice packings mentioned above, which are not uniformly stable, are nevertheless finitely stable.
\end{abstract}

* The first two authors were partially supported by the Hungarian National Science Foundation, Grant Numbers 326-0413 and A-221/95. 


\section{Definitions and Terminologies}

\subsection{Definitions Concerning Sphere Packings}

Many of the notions we use here were first introduced by L. Fejes Tóth in [F1] and [F2] and there are some taken from [C1] and [C2]. In this paper we will discuss arrangements of $d$-dimensional balls in the Euclidean $d$-dimensional space $E^{d}$. A ball packing (also called a sphere packing) in $E^{d}$ is an arrangement of $d$-dimensional closed balls which have disjoint interiors.

In order to describe how firm or steady a sphere packing as a structure is, we introduce the following notions. A sphere packing $\mathcal{P}$ is said to be:

(i) Stable (also 1-stable) if each ball is fixed by its neighbors.

(ii) $n$-Stable if each set of $n$ balls is fixed by its neighbors.

(iii) Finitely-stable if it is $n$-stable for every $n \geq 1$.

(iv) Uniformly stable if there is an $\varepsilon>0$, such that no finite subset of the balls of $\mathcal{P}$ can be rearranged such that each ball is moved by a distance less than $\varepsilon$ and the rearranged balls together with the rest of the balls form a packing different from $\mathcal{P}$.

Let us point out quickly that it is an easy exercise to show that the following is an equivalent condition for finite stability of sphere packings of $E^{d}$.

(iii*) A sphere packing $\mathcal{P}$ of $E^{d}(d>1)$ is finitely stable if and only if for any finite subset $\mathcal{P}^{\prime}$ of the balls of $\mathcal{P}$, there exists an $\varepsilon_{\mathcal{P}^{\prime}}>0$ such that one cannot rearrange the balls of $\mathcal{P}^{\prime}$ such that each ball is moved by a distance less than $\varepsilon_{\mathcal{P}^{\prime}}$ and the rearranged balls together with the rest of the packing form a packing different from $\mathcal{P}$.

Thus the different stability properties of sphere packings were listed in strengthening order, i.e., (iv) $\Rightarrow$ (iii) $\Leftrightarrow$ (iii*) $\Rightarrow$ (ii) $\Rightarrow$ (i).

\subsection{Delaunay Tiling}

Let $\mathcal{P}$ be a sphere packing of $E^{d}$ with unit balls. Let $C$ be the family of center points of the balls of $\mathcal{P}$. Consider a $d$-dimensional ball whose interior does not contain any center point from $C$ and whose boundary intersects $C$ in a set spanning $E^{d}$. The convex hull of the intersection of the $d$-dimensional ball with $C$ is a Delaunay cell. It turns out that for packings of congruent spheres not all contained in a half-space, such as we consider here, the Delaunay cells form a tiling $D$ of $E^{d}$, the Delaunay tiling.

\subsection{Frameworks}

The following definitions are taken from [RW]. Let $G$ be a finite or infinite graph with no loops or multiple edges. We will refer to the vertices of $G$ by $1,2, \ldots$ Each edge 
is labeled either a rod, cable, or a strut. Each vertex is labeled either fixed or variable. A realization of $G$ in $E^{d}$ is an assignment of a point $p_{i}$ in $E^{d}$, for the $i$ th vertex of $G$, $i=1,2, \ldots$. There is no restriction about edges crossing each other. A realization of $G$ is denoted by $G(p)$ where $p=\left(p_{1}, p_{2}, \ldots\right) . G(p)$ is called a (tensegrity) framework.

Let $p(t)=\left(p_{1}(t), p_{2}(t), \ldots\right)$, where $p_{i}(t)$ is continuous for $0 \leq t \leq 1, p(0)=p$, and $p_{i}(t)=p_{i}$, for the fixed vertices $i$ of $G$, for all $t$. We say $p(t)$ is a continuous motion or a flex of $G(p)$ if cables are not increased, the rods are not changed, struts are not decreased in length, and fixed vertices remain constant during the flex. If $G(p)$ admits only the identity flex, then we say $G(p)$ is rigid.

A framework $G(p)$ with infinitely many vertices is said to be finitely rigid if labeling all but finitely many vertices fixed always results in a rigid framework. This is the same as saying that the framework does not allow any motion once all, but finitely many, vertices are fixed.

An equilibrium stress $\omega=\left(\ldots, \omega_{i j}, \ldots\right)$ of the finite framework $G(p)$ is an assignment of a scalar $\omega_{i j}=\omega_{j i}$ for each edge $\{i, j\}$ of $G$ such that the following equilibrium equation holds for each variable vertex $i$ of $G$

$$
\sum_{j} \omega_{i j}\left(p_{i}-p_{j}\right)=0
$$

This vector sum is taken over all vertices $j$ adjacent to $i$. A proper equilibrium stress is an equilibrium stress $\omega$ such that $\omega_{i j}=\omega_{j i} \geq 0$ if $\{i, j\}$ is a cable, and $\omega_{i j}=\omega_{j i} \leq 0$ if $\{i, j\}$ is a strut (no condition on rods). Note that there is no equilibrium condition for fixed vertices.

\subsection{Outline of the Rest of the Paper}

One of the purposes of this paper is to show the virtues of the property (iv), uniform stability, and to show that it is strictly stronger than finite stability (iii). In Section 2 we develop a method that can be used to check whether some sphere packings are uniformly stable. Among others we show that the lattice packings $D_{d}$ and $A_{d}$ for $d \geq 3$ in $E^{d}$ are all uniformly stable. In Section 3 we give a constructive proof to show that the densest cubic lattice packing of $E^{d}, d \geq 2$, with unit balls, is not uniformly stable. In Section 4 we show that this packing $i$ f finitely stable, while at the same time we develop necessary and sufficient conditions for finite stability as in [C1]. (Note that some similar results concerning stabilities of sphere coverings are proved in [BBC].)

We believe that uniform stability is a natural and useful setting for the local stability of packings of spheres. At first sight it might seem that finite stability would be sufficient. However, imagine a box packed with hard spherical balls in contact, where the centers form a part of a cubical lattice. Mathematically there is no continuous motion that will allow the balls to move; but even the smallest amount of deformability will allow the whole configuration to collapse, when the number of balls is large enough.

Indeed, when the Delaunay cells of the packing satisfy the conditions of Theorem 2.7.1 (stated later, where the Delaunay cells can only increase in volume, since the packing condition constrains the vertices from getting any closer), then not only is the packing uniformly stable, but there is no local process that would increase its density as well. 
In [R] Reynolds discusses a property of packings, where the packing density (of a finite packing with respect to a container) decreases as the packing is perturbed while the container maintains external pressure. He calls this property "dilatancy." When the Delaunay cells satisfy the volume expanding property, we have a precise explanation of the packing's dilatancy. However, it is not clear whether this property will hold for other more complicated packings. There may be some need for more than one Delaunay cell in order to provide the needed rigidity.

\section{Uniform Stability and Volume}

\subsection{Volume}

In what follows we are interested in how the volume of a polytope $P$ changes as we perturb its vertices. If $P$ is simplicial, the perturbed polytope is easy to define. When $P$ is not simplicial there is an extra complication. We deal with this by first subdividing $P$ by inserting a vertex at the barycenter of each face of $P$, which enables us to triangulate the underlying space of $P$. When the vertices of $P$ are perturbed we consider the volume of this underlying simplicial complex $K$. This has the advantage that $K$ reflects the symmetries of $P$, when they exist. Also, when $P$ is part of a tiling, the simplicial complexes still form a tiling, because perturbed adjacent faces are subdivided consistently.

Definition 2.1.1. Let $P$ be a convex polytope in $E^{d}$ with vertices $p_{1}, \ldots, p_{n}$. Let $F=\left\langle q_{1}, \ldots, q_{m}\right\rangle$ be a face of $P$. The barycenter of $F$ is

$$
\hat{F}=\frac{1}{m} \sum_{i=1}^{m} q_{i}
$$

Let $F_{0} \subset F_{1} \subset \cdots \subset F_{k}$ denote a sequence of faces, called a flag, of $P$, where $F_{0}$ is a vertex and $F_{i-1}$ is a facet (a face one dimension lower) of $F_{i}$ for $i=1, \ldots, k$. Then the simplices of the form

$$
\left\langle\hat{F}_{0}, \hat{F}_{1}, \ldots, \hat{F}_{k}\right\rangle
$$

constitute a simplicial complex $K$ whose underlying space is the boundary of $P$. See $[\mathrm{H}]$ or $[\mathrm{RS}]$ for a discussion of this complex.

We regard all points in $E^{d}$ as row vectors and use $q^{T}$ for the column vector that is the transpose of the row vector $q$. Moreover, $\left[q_{1}, \ldots, q_{d}\right]$ is the matrix with the $i$ th row $q_{i}$.

Choosing a $(d-1)$-simplex of the simplicial complex $K$ to be positively oriented, one can generate a positive orientation for all $(d-1)$-simplices of $K$. For each $(d-1)$ simplex $\left\langle\hat{F}_{0}, \hat{F}_{1}, \ldots, \hat{F}_{d-1}\right\rangle$ let $\operatorname{pos}\left\langle\hat{F}_{0}, \hat{F}_{1}, \ldots, \hat{F}_{d-1}\right\rangle$ denote any permutation of the vertices $\hat{F}_{0}, \hat{F}_{1}, \ldots, \hat{F}_{d-1}$ that corresponds to a positive orientation of the $(d-1)$ simplex $\left\langle\hat{F}_{0}, \hat{F}_{1}, \ldots, \hat{F}_{d-1}\right\rangle$ in the simplicial complex $K$. Finally, let $\operatorname{sign}\left\langle\hat{F}_{0}, \ldots, \hat{F}_{d-1}\right\rangle$ be equal to 1 (resp. -1$)$ if the orientations of the $(d-1)$-simplices $\operatorname{pos}\left\langle\hat{F}_{0}, \hat{F}_{1}, \ldots, \hat{F}_{d-1}\right\rangle$, $\left\langle\hat{F}_{0}, \hat{F}_{1}, \ldots, \hat{F}_{d-1}\right\rangle$ are the same (resp. different) in the simplicial complex $K$. Then the following is clear. 
Lemma 2.1.1. The signed volume of $P$ is

$$
V(P)=\frac{1}{d !} \sum_{F_{0} \subset \cdots \subset F_{d-1}} \operatorname{sign}\left\langle\hat{F}_{0}, \ldots, \hat{F}_{d-1}\right\rangle \operatorname{det}\left[\hat{F}_{0}, \ldots, \hat{F}_{d-1}\right]
$$

where the sum is taken over all flags of faces $F_{0} \subset F_{1} \subset \cdots \subset F_{d-1}$ of $P$, and $\operatorname{det}[]$ is the determinant function.

Here it is understood that the orientation is chosen so that the volume is positive. (It is an easy exercise to show that the quantity defined by (2.1.3) is independent of the choice of the origin, and that it agrees with the usual definition of volume.)

Later it will help to rewrite (2.1.3) in terms of the wedge product of vectors. So

$$
\operatorname{det}\left[\hat{F}_{0}, \hat{F}_{1}, \ldots, \hat{F}_{d-1}\right]=\hat{F}_{0} \wedge \hat{F}_{1} \wedge \cdots \wedge \hat{F}_{d-1} .
$$

See $[\mathrm{S}]$ for a definition of the wedge product of vectors.

\subsection{Infinitesimal Motions}

We wish to compute the gradient of the functional $V(P)$, where $P$ is regarded as a function of its vertices. To do this we consider an arbitrary path $p(t)=p+t p^{\prime}$ in the space of configurations of the vertices $p=\left(p_{1}, \ldots, p_{n}\right)$, where $p^{\prime}=\left(p_{1}^{\prime}, \ldots, p_{n}^{\prime}\right)$. We then compute the derivative of $V\left(P_{t}\right)$ in the direction $p^{\prime}$, where $P_{t}$ is the polytope whose underlying space is triangulated by the simplicial complex $K_{t}$ whose vertices are defined from $p(t)$ by (2.1.1) and whose simplices are defined by (2.1.2). We compute:

$V\left(P_{t}\right)=\frac{1}{d !} \sum_{F_{0} \subset F_{1} \subset \cdots \subset F_{d-1}} \operatorname{sign}\left\langle\hat{F}_{0}(t), \hat{F}_{1}(t), \ldots, \hat{F}_{d-1}(t)\right\rangle \hat{F}_{0}(t) \wedge \hat{F}_{1}(t) \wedge \cdots \wedge \hat{F}_{d-1}(t)$,

where the sum is taken over all flags of length $d$ of $P$ as before, and

$$
\hat{F}_{i}(t)=\frac{1}{m} \sum_{i=1}^{m} q_{i}(t)=\frac{1}{m} \sum_{i=1}^{m}\left(q_{i}+t q_{i}^{\prime}\right) .
$$

Then

$\frac{d}{d t} V\left(P_{t}\right)=\frac{1}{d !} \sum_{F_{0} \subset \cdots \subset F_{d-1}} \operatorname{sign}\left\langle\hat{F}_{0}(t), \ldots, \hat{F}_{d-1}(t)\right\rangle \sum_{i=0}^{d-1} \hat{F}_{0}(t) \wedge \cdots \wedge \frac{d \hat{F}_{i}(t)}{d t} \wedge \cdots \wedge \hat{F}_{d-1}(t)$.

Evaluating this derivative at $t=0$, collecting terms, and using the anticommutativity of the wedge product we get:

$$
V^{\prime}=\left.\frac{d}{d t} V\left(P_{t}\right)\right|_{t=0}=\frac{1}{d !} \sum_{i} N_{i} \wedge p_{i}^{\prime}
$$

where each $N_{i}$ is some linear combination of wedge products of $(d-1)$ vectors $p_{j}$, where $p_{j}$ and $p_{i}$ share a common face. Figure 1 shows the way in which the polytope $P_{t}$ 

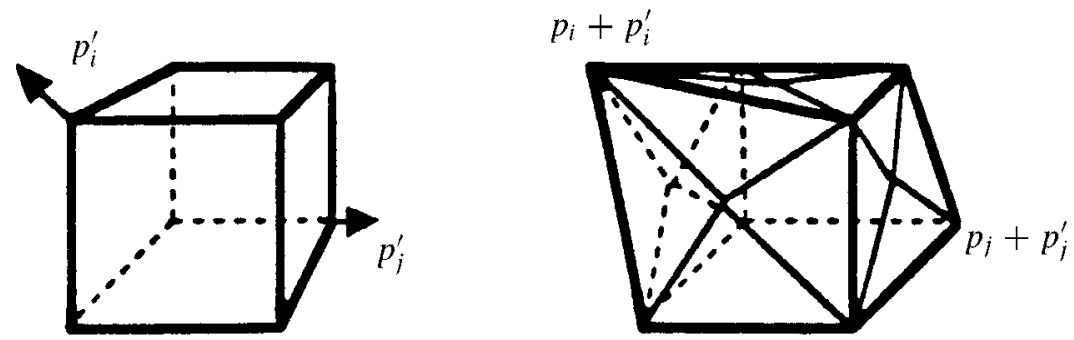

Fig. 1

deforms under the motion given by $p^{\prime}$. We have indicated the barycenters of the square faces only in the deformed polyhedron. This is because for the edges (and any face that is a simplex), the barycenter is chosen in the line determined by the edge itself (or in the convex hull of the deformed simplex). Thus the actual set occupied by the deformed polyhedron does not have that barycenter as a vertex. So in order not to clutter the picture, we have left those barycenters out.

Definition 2.2.1. We call $N=\left(N_{1}, \ldots, N_{n}\right)$ the volume force of $P$.

The following are some properties of $V^{\prime}$.

(1) Each $N_{i}$ is only a function of the vertices that share a face (or facet) with $p_{i}$ but not $p_{i}$ itself. (To see this rewrite the formula for $\hat{F}_{i}(t)$ before differentiating in such a way that no $q_{i}$ is repeated in any term, using the anticommutativity.)

(2) Let $T: E^{d} \rightarrow E^{d}$ be a congruence of $E^{d}$ that is invariant on $P$. That is, $T(P)=P$ and $T$ permutes the vertices of $P$. Suppose $T\left(p_{i}\right)=p_{j}$. Then $T\left(N_{i}\right)=N_{j}$. Note also that if $i=j$ and $T\left(p_{i}\right)=p_{i}$, then $T\left(N_{i}\right)=N_{i}$. In other words $N_{i}$ is in the fixed set of $T$. (We regard 0 as the barycenter of $P$, so $T$ is an orthogonal linear function on $E^{d}$.)

\subsection{The Constraining Graph}

Suppose $P$ is a convex polytope in $E^{d}$ as before with vertices $p=\left(p_{1}, \ldots, p_{n}\right)$. Let $G$ be a graph defined on this vertex set $p$. In our applications, $G$ may or may not consist of edges of $P$. We think of the edges of $G$ as defining those pairs of vertices that are constrained not to get closer. In the terminology of the geometry of rigid tensegrity frameworks each edge of $G$ is a strut.

Let $p^{\prime}=\left(p_{1}^{\prime}, \ldots, p_{n}^{\prime}\right)$ be an infinitesimal flex of $G(p)$. That is for each strut $\{i, j\}$ of $G$ we have

$$
\left(p_{i}-p_{j}\right) \cdot\left(p_{i}^{\prime}-p_{j}^{\prime}\right) \geq 0 .
$$

We can rewrite these inequalities in terms of a single rigidity matrix $R(p)=\frac{1}{2} d f_{p}$, where $f(p)=\left(\ldots,\left|p_{i}-p_{j}\right|^{2}, \ldots\right) \in E^{e}$ and where $e$ is equal to the number of edges 
of $G$, as follows:

$$
R(p)\left(p^{\prime}\right)^{T}=\left(\begin{array}{c}
\vdots \\
\left(p_{i}-p_{j}\right) \cdot\left(p_{i}^{\prime}-p_{j}^{\prime}\right) \\
\vdots
\end{array}\right) \geq 0,
$$

where the inequality is meant for each coordinate.

We say that the infinitesimal motion $p^{\prime}=\left(p_{1}^{\prime}, \ldots, p_{n}^{\prime}\right)$ is strictly volume increasing if in addition to (2.3.1) we have

$$
V^{\prime}=\frac{1}{d !} \sum_{i} N_{i} \wedge p_{i}^{\prime}>0
$$

(or we say $p^{\prime}$ is volume preserving if (2.3.2) is equality). Here $N_{i}$ is regarded as a $d$ dimensional vector so that $N_{i} \wedge p_{i}^{\prime}$ can be interpreted as the standard inner product $N_{i} \cdot p_{i}^{\prime}$, with appropriate identification of bases.

For each edge $\{i, j\}$ of $G$, let $\omega_{i j}$ be a scalar. We collect all such scalars into a single row vector called a stress $\omega=\left(\ldots, \omega_{i j}, \ldots\right)$ corresponding to the rows of the matrix $R(p)$. Append one more row onto $R(p)$ to get a new matrix $\tilde{R}(p)$, which we call the augmented rigidity matrix, so that

$$
\tilde{R}(p)\left(p^{\prime}\right)^{T}=\left(\begin{array}{c}
\vdots \\
\left(p_{i}-p_{j}\right) \cdot\left(p_{i}^{\prime}-p_{j}^{\prime}\right) \\
\vdots \\
\sum_{i} N_{i} \cdot p_{i}^{\prime}
\end{array}\right) .
$$

We calculate:

$$
(\omega, 1) \tilde{R}(p)=\left(\ldots, \sum_{j} \omega_{i j}\left(p_{i}-p_{j}\right)+N_{i}, \ldots\right),
$$

where each sum is taken over all $p_{j}$ adjacent in $G$ to $p_{i}$, and we collect $d$ coordinates at a time.

Definition 2.3.1. Let $N=\left(N_{1}, \ldots, N_{n}\right)$ be any sequence of vectors assigned to the vertices $p_{i}$ of $P$ ( $N$ not necessarily the volume force). We say the stress $\omega$ resolves $N$ (regarded as a force) if for each $i$

$$
\sum_{j} \omega_{i j}\left(p_{i}-p_{j}\right)+N_{i}=0
$$

or, equivalently, $(\omega, 1) \tilde{R}(p)=0$.

The following Theorem is the main working tool for showing our stability results. The polytope $P$ mentioned can be any $(d-1)$-dimensional polyhedral manifold in $d$-space (or for that matter any polyhedral " $(d-1)$-homology cycle"), but for all the applications that we have in mind, it enough to consider only the case when $P$ is a convex polytope. 
Theorem 2.3.1. Suppose that the volume force $N$ of a polytope $P$ can be resolved by a stress $\omega$ on the strut-framework $G(p)$ such that for each edge $\{i, j\}$ of $G, \omega_{i j}<0$, where $G$ is a graph on the vertices of $P$. Let $p^{\prime}=\left(p_{1}^{\prime}, \ldots, p_{n}^{\prime}\right)$ be any infinitesimal motion on $G(p)$. Then the infinitesimal change in the volume, $V^{\prime} \geq 0$, and equality occurs if and only if $\left(p_{i}-p_{j}\right) \cdot\left(p_{i}^{\prime}-p_{j}^{\prime}\right)=0$ for each edge $\{i, j\}$ of $G$.

Proof. We calculate using the associativity of matrix multiplication and the inequality (2.3.1):

$$
0=0 \cdot p^{\prime}=(\omega, 1) \tilde{R}(p)\left(p^{\prime}\right)^{T}=\sum_{i j} \omega_{i j}\left(p_{i}-p_{j}\right)\left(p_{i}^{\prime}-p_{j}^{\prime}\right)+\sum_{i} N_{i} \cdot p_{i}^{\prime} \leq V^{\prime} .
$$
of $G$.

We clearly get equality if and only if $\left(p_{i}-p_{j}\right) \cdot\left(p_{i}^{\prime}-p_{j}^{\prime}\right)=0$ for all $\{i, j\}$ edges

With this theorem in mind we say the following.

Definition 2.3.2. The convex polytope $P$ and the graph $G$ on the vertices of $P$ satisfy the critical volume condition if the volume force $N$ can be resolved by a stress $\omega=$ $\left(\ldots, \omega_{i j}, \ldots\right)$ on the edges of $G$ so that for each edge $\{i, j\}$ of $G, \omega_{i j}<0$.

We will compute several examples of polytopes and graphs with and without the critical volume condition in the next sections.

\subsection{Examples with the Critical Volume Condition}

Example 2.4.1. We consider first the case of the plane where "volume" becomes area, and the polytope $P$ becomes a convex polygon. We first consider only deformations of $P$ that preserve the lengths of edges of $P$. It is known that the area function is critical if and only if the vertices of the polygon either lie on a circle or a line. See [BG] for information about this fact. Assume $P$ satisfies the critical volume condition. By Theorem 2.3.1, $V^{\prime} \geq 0$ for any first-order motion on $G(p)$. Restricted to infinitesimal motions which satisfy $\left(p_{i}-p_{j}\right)\left(p_{i}^{\prime}-p_{j}^{\prime}\right)=0$ for all edges of the polygon, Theorem 2.3.1 gives $V^{\prime}=0$. This means that $p$ is a critical point of the area function.

We leave it as an exercise for the reader to check that when the vertices of $P$ lie on a circle $C, P$ satisfies the critical area condition if and only if the center of $C$ lies in the interior of $P$. Figure 2 is a diagram of the area force for a pentagon. (Notice that due to the proper identification of bases of the vectors $N_{i}$ and points in $E^{2}$ each vector of the area force is perpendicular to the proper diagonal of the pentagon pointing to the exterior of the pentagon.)

Example 2.4.2. In any dimension, let $P$ be a simplex and the graph $G$ all pairs of vertices, then $P$ has the critical volume condition if and only if the orthogonal projection of each vertex lies in the interior of the opposite facet. In the plane this means $P$ is an acute triangle. In $E^{3}$ this means $P$ has all dihedral angles acute. Etc. 


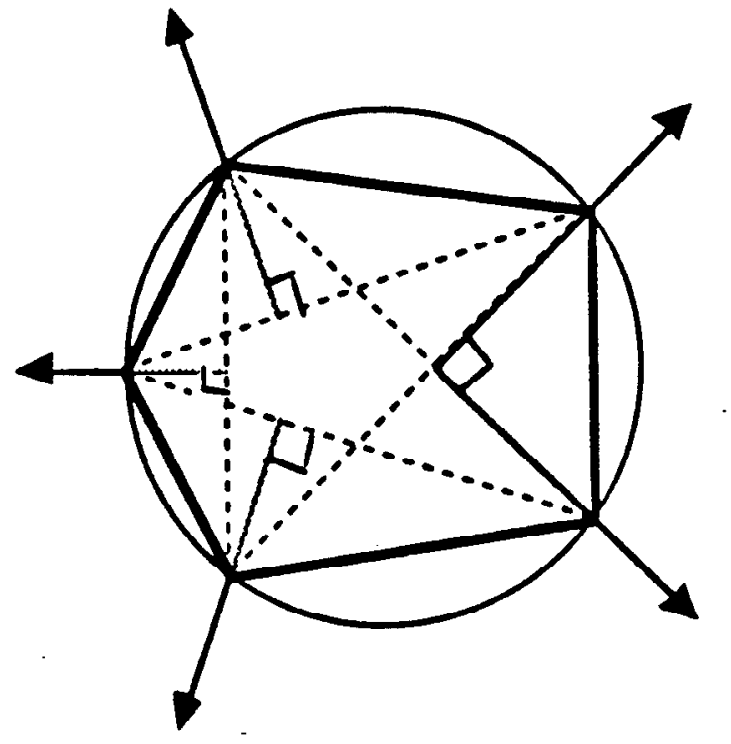

Fig. 2

Example 2.4.3. In $E^{3}$ the regular octahedron $P$ has a volume force $N_{i}$ that is a scalar multiple of $p_{i}$, where $\left(p_{1}, \ldots, p_{8}\right)$ are the vertices of $P$ and the origin is at the center of $P$. When the graph $G$ is the set of edges of $P$, the volume force is resolved by a stress that has all the same values on each edge.

\subsection{Infinitesimal Rigidity}

We briefly review some basic definitions. See [C2] for more information. Consider now just the bar graph $\bar{G}$, which is the graph $G$ with all the struts changed to bars, and

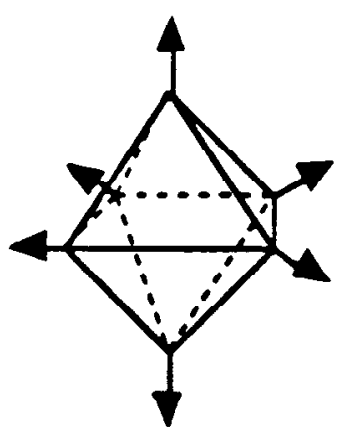

Fig. 3 
its configuration $p=\left(p_{1}, \ldots, p_{n}\right)$ in $E^{d}$ to get a bar framework $\bar{G}(p)$. We say that infinitesimal motion $p^{\prime}=\left(p_{1}^{\prime}, \ldots, p_{n}^{\prime}\right)$ is an infinitesimal flex of $\bar{G}(p)$ if for all $\{i, j\}$ is an edge of $\bar{G}$, we have

$$
\left(p_{i}-p_{j}\right) \cdot\left(p_{i}^{\prime}-p_{j}^{\prime}\right)=0 .
$$

This is the same as saying $R(p)\left(p^{\prime}\right)^{T}=0$ for the rigidity matrix $R(p)$.

Definition 2.5.1. We say $p^{\prime}$ is a trivial infinitesimal flex if $p^{\prime}$ is the (directional) derivative of a congruence of $E^{d}$. We say that $G(p)$ (resp. $\left.\bar{G}(p)\right)$ is infinitesimally rigid if $G(p)($ resp. $\bar{G}(p))$ has only trivial infinitesimal flexes.

The following combines (infinitesimal) rigidity and the critical volume condition.

Corollary 2.5.1 to Theorem 2.3.1. Suppose a convex polytope $P$ and a graph $G$ on the vertices of $P$ satisfy the critical volume condition and $\bar{G}(p)$ (as a bar framework) is infinitesimally rigid. Then $V^{\prime}>0$ for every nontrivial infinitesimal flex $p^{\prime}$ of the tensegrity framework $G(p)$.

Proof. By Theorem 2.3.1, $V^{\prime} \geq 0$. If $V^{\prime}=0$, then $p^{\prime}$ is an infinitesimal flex of the bar graph $\bar{G}(p)$. However, then by the infinitesimal rigidity of $\bar{G}(p)$, this would imply $p^{\prime}$ is trivial. Thus $V^{\prime}>0$.

It is a general principle that infinitesimal rigidity implies the "rigidity" of a framework. In [C2] (see also [CW]) this is discussed in the context of second-order rigidity. Similar principles apply in our situation to the volume function.

Corollary 2.5.2 to Theorem 2.3.1. Suppose a convex polytope $P$ and a graph $G$ on the vertices of $P$ satisfy the critical volume condition and $\bar{G}(p)$ is infinitesimally rigid. Then there is an $\varepsilon>0$ with the following property. If $q=\left(q_{1}, \ldots, q_{n}\right)$ is another configuration of the vertices of $P$ with

$$
\begin{array}{ll}
\left|p_{i}-q_{i}\right|<\varepsilon & \text { for all } i=1, \ldots, n, \\
\left|p_{i}-p_{j}\right| \leq\left|q_{i}-q_{j}\right| & \text { for edges } \quad\{i, j\} \text { of } G,
\end{array}
$$

then $V(P) \leq V(Q)$ with equality only when $P$ is congruent to $Q$, where $Q$ is the polytope determined by the vertices $q$, and the points defining simplices by (2.1.1).

Proof. The inequalities (2.5.2) define a semialgebraic set $X$ in the space of all configurations $\left\{\left(q_{1}, \ldots, q_{n}\right) \mid q_{i} \in E^{d}, i=1, \ldots, n\right\}$. Suppose there is no $\varepsilon>0$ as in the conclusion. Add $V(P) \geq V(Q)$ to the constraints defining $X$. By Wallace [Wa] (see [C2]) there is an analytic path $p(t)=\left(p_{1}(t), \ldots, p_{n}(t)\right), 0 \leq t<1$, with $p(0)=p$ and $p(t) \in X, p(t)$ not congruent to $p(0)$ for $0<t<1$. So

$$
\begin{aligned}
\left|p_{i}-p_{j}\right| & \leq\left|p_{i}(t)-p_{j}(t)\right| & & \text { for }\{i, j\} \text { an edge of } G \text { and } \\
V(P) & \geq V\left(P_{t}\right) & & \text { for } 0 \leq t \leq 1 .
\end{aligned}
$$


Then after suitably adjusting $p(t)$ by congruences (as in [C2] as well as [CW]) we can define

$$
p^{\prime}=\left.\frac{d^{k} p(t)}{d t^{k}}\right|_{t=0},
$$

for the smallest $k$ that makes $p^{\prime}$ a nontrivial infinitesimal flex. (Such a $k$ exists by the argument in [C2] as well as [CW]).

Since (2.5.3) holds we see that $p^{\prime}$ is a nontrivial infinitesimal flex of $G(p)$, and

$$
V^{\prime}=\left.\frac{d^{k} V\left(P_{t}\right)}{d t^{k}}\right|_{t=0} \leq 0,
$$

is the infinitesimal change in the volume corresponding to $p^{\prime}$. But this contradicts Corollary 2.5.1 to Theorem 2.3.1. So $\varepsilon>0$ as in (2.5.1) exists such that the conclusion follows.

With the above result in mind, let $P$ be a polytope in $E^{d}$ and let $G$ be a graph on the vertices $p=\left(p_{1}, \ldots, p_{n}\right)$ of $P$.

Definition 2.5.2. We say $P, G$ is strictly locally volume expanding if there is an $\varepsilon>0$ such that for every $q$ satisfying (2.5.1) and (2.5.2) we have $V(P) \leq V(Q)$ with strict inequality unless $P$ is congruent to $Q$.

\subsection{More Examples}

In Section 2.4 we discussed some examples of polytopes with the critical volume condition. We calculate which of those examples also has an infinitesimally rigid bar framework.

Example 2.4.1. This is a polygon $P$ in the plane where all its vertices lie on a circle. If we take the graph $G$ to be the edges of $P, \bar{G}(p)$ is infinitesimally rigid and $P, G$ is strictly locally volume expanding if and only if $P$ is an acute triangle.

Example 2.4.2. This is a simplex $P$ in $E^{d}$. We take $G$ to be all the edges of $P$. Then $\bar{G}(p)$ will always be infinitesimally rigid. So $P, G$ is strictly locally volume expanding when each vertex projects into the interior of the opposite facet.

Example 2.4.3. In $E^{d}$ the regular octahedron $P$ satisfies the critical volume condition. We take $G$ to be all the edges of $P$. It is a classical theorem (see Dehn [D] and Gluck [Gl] for an easy proof) that $\bar{G}(p)$ is infinitesimally rigid. Thus $P, G$ is strictly locally volume expanding.

In what follows we will need a series of examples that will generalize Examples 2.4.2 and 2.4.3 above. Let $1 \leq k<d$ be positive integers. Let $D(k, d)$ be the convex hull of 
those vectors in $E^{d}$ that have exactly $k$ entries that are 1 , and $d-k$ entries that are 0 . Alternately we see that

$$
D(k, d)=[0,1]^{d} \cap\left\{\left(x_{1}, \ldots, x_{d}\right) \mid \sum_{i=1}^{d} x_{i}=k\right\},
$$

where $[0,1]^{d}$ is the unit cube in $E^{d}$, and $[0,1]^{d}=\left\{\left(x_{1}, \ldots, x_{d}\right) \mid 0 \leq x_{i} \leq 1\right.$ for all $i=$ $1, \ldots, d\}$. Thus we regard $D(k, d)$ as a subset of a $(d-1)$-dimensional hyperplane.

There are two kinds of facets of $D(k, d)$. Let $H_{i}=\left\{\left(x_{1}, \ldots, x_{d}\right) \mid x_{i}=0\right\}$, and let $H^{i}=\left\{\left(x_{1}, \ldots, x_{d}\right) \mid x_{i}=1\right\}$. Then for any $i=1, \ldots, d, D(k, d) \cap H_{i}$ is the convex hull of these vertices in $D(k, d)$ whose $i$ th coordinate is 0 . Thus $D(k, d) \cap H_{i}$ is congruent to $D(k, d-1)$, as long as $k<d-1$ and is simply one point if $k=d-1$. Similarly for any $i=1, \ldots, d, D(k, d) \cap H^{i}$ is the convex hull of those vertices of $D(k, d)$ whose $i$ th coordinate is 1 . Thus $D(k, d) \cap H_{i}$ is congruent to $D(k-1, d-1)$ as long as $k>1$ and is simply one point when $k=1$.

We see that $D(1, d)$ is an $(d-1)$-dimensional regular simplex, and $D(2,4)$ is a regular octahedron, since it consists of the convex hull of $\left(\begin{array}{l}4 \\ 2\end{array}\right)=6$ vertices, where all pairs of these points are $\sqrt{2}$ apart except for three disjoint pairs.

We also observe that the edges of any $D(k, d)$ are all $\sqrt{2}$ in length. Two vertices belong to the same edge if and only if they differ in exactly two coordinates and thus are $\sqrt{2}$ distance apart. Notice also $D(k, d)$ is congruent to $D(d-k, d)$.

Example 2.6.1. In $E^{d-1}$ we take the polytope to be $D(k, d)$ for $1 \leq k<d$. Any permutation of the $d$-coordinates provides a symmetry of $D(k, d)$, a congruence to itself, by permuting the coordinates. Thus for any given vertex $p_{i}$ of $D(k, d)$, the group of symmetries of $D(k, d)$, fixing $p_{i}$, fix only the line through the center of $D(k, d)$ and $p_{i}$. Thus $D(k, d)$ has the critical volume condition.

Let $G$ be the graph determined by those vertices of $D(k, d)$ that are $\sqrt{2}$ apart, the edges of $D(k, d)$.

Lemma 2.6.1. The bar framework $\bar{G}(p)$ is infinitesimally rigid in $E^{d-1}$.

Proof. Inductively, each $D(1, d), D(d-1, d)$, and $D(2,4)$ all have their corresponding bar frameworks infinitesimally rigid, as discussed in previous examples. For $d \geq 5$, $k=1, \ldots, d-1$, each facet of $D(k, d)$ has as its corresponding bar framework another $D(k-1, d-1)$ or $D(k, d-1)$ which we can assume is infinitesimally rigid in their hyperplane of dimension $d-2$, by induction. It is known (see [W], for example) that if all the facets of a convex polytope $P$ in $E^{d-1}$ are infinitesimally rigid in the hyperplane of their facet, for $d \geq 3$, then the whole graph is infinitesimally rigid. Thus the bar framework of $D(k, d)$ is infinitesimally rigid in $E^{d-1}$. Alternatively, it is also easy to see that the 2-skeleton (the collection of two-dimensional faces) of $D(k, d)$ is triangulated for $1 \leq k \leq d$. Then by a theorem of Whiteley [W] it is known that the 1 -skeleton is infinitesimally rigid as a bar graph.

Then $D(k, d), G$ for $1 \leq k<d$ is strictly locally volume expanding. 
Remark. The polytope $D(2, d), d \geq 4$ in $E^{d-1}$ has exactly

$$
\left(\begin{array}{l}
d \\
2
\end{array}\right)=\frac{d(d-1)}{2}
$$

vertices and

$$
\frac{1}{2} \frac{d(d-1)}{2} \cdot 2(d-2)=\frac{d(d-1)(d-2)}{2}
$$

edges. In order for a bar framework with $v$ vertices and $e$ edges to be infinitesimally rigid in $E^{d}$ we must have

$$
e \geq d v-\frac{d(d+1)}{2}
$$

In our case

$$
e=\frac{d(d-1)(d-2)}{2}=\frac{(d-1) d(d-1)}{2}-\frac{(d-1) d}{2}=(d-1) v-\frac{(d-1) d}{2} .
$$

Thus each edge is needed with no redundancy.

\subsection{Tilings and Packings}

Suppose the $\mathcal{P}$ is a packing of spherical balls in $E^{d}$. Let $G$ be the graph of $\mathcal{P}$, where the centers of the balls serve as the vertices of $G$ and an edge is placed between two vertices when the corresponding two balls intersect. The following is a basic principle that can be used to show that many packings $\mathcal{P}$ are uniformly stable.

Theorem 2.7.1. Suppose $E^{d}$ can be tiled face-to-face by congruent copies of finitely many convex polytopes $P_{1}, \ldots, P_{m}$, such that each $P_{i}$ and $G$ restricted to the vertices of each $P_{i}$ is strictly locally volume expanding. Then the packing $\mathcal{P}$ is uniformly stable.

Proof. Let $\varepsilon>0$ be the minimum for each $P_{i}$ and $G$ restricted to $P_{i}$ as guaranteed by the strict locally volume expanding property. All but a finite number of the tiles are fixed. The tiles that are free to move are confined to a region of fixed volume in $E^{d}$. Since each $P_{i}$ is strictly locally volume expanding, the volume of each of the tiles must be fixed. But the strict condition implies that the motion on each tile must be a congruence. Since the tiling is face-to-face and the vertices are given by $G$ we conclude inductively (on the number of tiles) that each vertex of $G$ must be fixed. Thus $\mathcal{P}$ is uniformly stable.

Let $\Lambda$ be a lattice in $E^{d}$, generated, say, by $g_{1}, \ldots, g_{d} \in E^{d}$, and let $\mathcal{P}$ be packing (of balls, say) in $E^{d}$. We say that $\mathcal{P}$ is periodic with respect to $\Lambda$, if for every $B \in \mathcal{P}$ and $\lambda \in \Lambda$, the translate of $B$ by $\lambda, B+\lambda$ is also in $\mathcal{P}$. Indeed, we say $\mathcal{P}$ is a finite packing of the quotient space $E^{d} / \Lambda$, if every element of $\mathcal{P}$ is a $\Lambda$ translate of one of a finite number of packing elements of $\mathcal{P}$, i.e., they generate $\mathcal{P}$. Note that if $\hat{\Lambda} \subset \Lambda$ is a sublattice, then 
there is a corresponding covering map

$$
\begin{gathered}
E^{d} / \hat{\Lambda} \\
\downarrow \\
E^{d} / \Lambda
\end{gathered}
$$

that also maps packing elements to packing elements.

We say that a periodic packing $\mathcal{P}$ with lattice $\Lambda$ is periodically stable if there is an $\varepsilon>0$, such that packing elements, $B_{1}, \ldots, B_{m} \in \mathcal{P}$ and generators $g_{1}, \ldots, g_{d}$ of $\Lambda$, we can guarantee that for any other periodic packing $\mathcal{P}$, generated by $B_{i}^{\prime} \varepsilon$-congruent to $B_{i}, i=1, \ldots, m$, periodic with respect to $\Lambda^{\prime}$ generated by $g_{1}^{\prime}, \ldots, g_{d}^{\prime},\left|g_{i}^{\prime}-g_{i}\right|<\varepsilon$, $i=1, \ldots, d$, such that

$$
\operatorname{Vol}\left(E^{d} / \Lambda\right) \geq \operatorname{Vol}\left(E^{d} / \Lambda^{\prime}\right)
$$

then $\Lambda$ is congruent to $\Lambda^{\prime}$ and $\mathcal{P}$ is congruent to $\mathcal{P}^{\prime}$.

Theorem 2.7.2. With a packing $\mathcal{P}$ as before, suppose $E^{d}$ can be tiled periodically with respect to $\Lambda$ face-to-face by congruent copies of finitely many convex polytopes $P_{1}, \ldots, P_{m}$, such that each $P_{i}$ is strictly volume expanding, with respect to $G$ the graph of the packing $\mathcal{P}$. Then $\mathcal{P}$ with respect to $\Lambda$ is periodically stable.

Proof. The idea is very similar to Theorem 2.7.1. Just observe that the strict volume expanding condition together with the volume condition for periodic stability combine to force the congruence.

Corollary 2.7.1. With the same conditions as in Theorem 2.7.2, and with $\hat{\mathcal{P}}$ a periodic subpacking of $\mathcal{P}$ given by a sublattice $\hat{\Lambda}$ of $\Lambda$, we still have periodic stability (with respect to the same $\varepsilon>0$.)

Remark 2.7.1. Notice that periodic stability is stronger than being locally maximal dense (see [C1] and [CS1]).

Remark 2.7.2. The techniques discussed above do not automatically provide an estimate for the $\varepsilon>0$ that is guaranteed by uniform stability. It is possible, at least in some cases (such as in Theorem 2.8.1 below), to extend our methods to find explicit estimates for $\varepsilon>0$, but these methods are more complicated and the effort does not seem to be worth it.

\subsection{A Uniformally Stable Packing in the Plane}

Consider the well-known triangular packing of circular disks of equal radii where each disk is adjacent to exactly six others. Remove one disk to obtain another packing $\mathcal{P}_{0}$. See Fig. 4 for the graph of $\mathcal{P}_{0}$. We state the following in our terminology. 


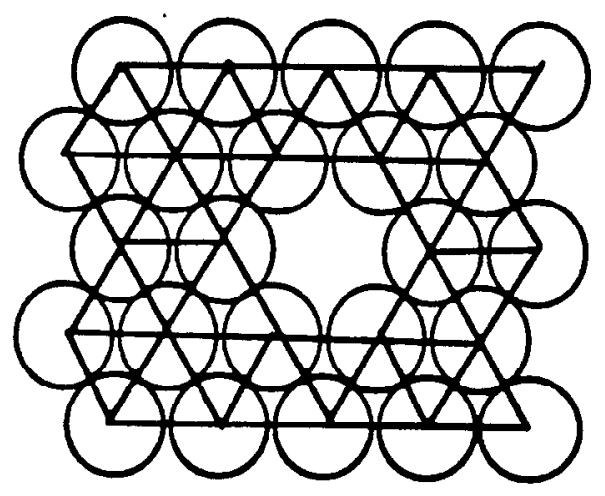

Fig. 4

Theorem 2.8.1 [BD]. The packing $\mathcal{P}_{0}$ is uniformally stable.

Proof. The graph of Fig. 4 describes a tiling of the plane into equilateral triangles (of side length $2 r$, where $r$ is the radius of the disks) and one regular hexagon (of side length $2 r$ ). Each triangle has the strict volume expanding condition. However, the hexagon does not. On the other hand, the hexagon does satisfy the critical area (two-dimensional volume) condition. (The underlying framework is not infinitesimally rigid though). But the hexagon is surrounded by triangles in such a way that the hexagon union on one layer, say as in Fig. 4, is infinitesimally rigid.

We can then use this polygon and the accompanying graph (with vertices inside) as in Theorem 2.7.1 to conclude that $\mathcal{P}_{0}$ is uniformly stable.

This proof is almost identical in outline to that of Bárány and Dolbilin. The only difference is in the calculation of the strict area increasing property.

Remark 2.8.1. An outstanding conjecture of L. Fejes Tóth says that the face incircles of a regular tiling $\{p, 3\}$ (that has precisely $\mathrm{p}$ regular triangles meeting at each vertex) with $p \geq 6$ form a strongly solid packing. That is, if we remove any circle from the packing then the packing of the remaining circles is solid. Recall that a circle packing is called solid if no finite subset of the circles can be rearranged such that the rearranged circles together with the rest of the circles form a packing not congruent to the original. (Obviously, any solid circle packing is uniformly stable.) The above conjecture of L. Fejes Tóth has been proved by Bezdek [B] for all $p \geq 8$. The open cases are the proper Euclidean circle packing for $p=6$ (the uniform stability of which is proved by Theorem 2.8.1) and the corresponding hyperbolic circle packing for $p=7$. 


\subsection{The Lattice Packings $D_{d}$ and $A_{d}$}

The lattice $D_{d}$ in $E^{d}$ is

$$
D_{d}=\left\{\left(x_{1}, \ldots, x_{d}\right) \mid \text { each } x_{i} \text { is an integer and } x_{1}+\cdots+x_{d} \text { is even }\right\},
$$

and the lattice $A_{d}$ in $E^{d}$ is

$$
A_{d}=\left\{\left(x_{1}, \ldots, x_{d+1}\right) \mid \text { each } x_{i} \text { is an integer and } x_{i}+\cdots+x_{d+1}=0\right\},
$$

where $E^{d}$ is regarded as the subspace of $E^{d+1}$ given by $x_{1}+\cdots+x_{d+1}=0$.

For both of these lattices there is a corresponding packing of congruent balls of radius $\sqrt{2} / 2$.

For any packing $\mathcal{P}$ of balls consider the configuration of centers $p=\left(p_{1}, \ldots p_{i}, \ldots\right)$ of packing elements. Let $S$ be any sphere that has a (locally) maximal radius with no points of $p$ in the interior of $S$. We call such an $S$ a Delaunay sphere. The convex hull of the vertices of $S$ is called a Delaunay cell.

It is a basic fact that the Delaunay cells of a (locally finite) point configuration form a face-to-face tiling of $E^{d}$.

According to Conway and Sloane [CS1, p. 144] the Delaunay cells of $D_{d}$ are of two types, $\beta_{d}$ the $d$-dimensional cross-polytope, and the "half-cube" $h \gamma_{d}$ which is the same as $D(2, d+1)$ in the notation of Section 2.6. Similarly, for the lattice packing $A_{d}$, the Delaunay cells are $D(k, d+1)$ for $k=1, \ldots, d$ (see [CS2] for an equivalent description, called "ambo-simplices," for those Delaunay cells). All of the polytopes with their edge graphs were shown to have the strict local volume expanding property (except $\beta_{d}$ which follows easily for $d \geq 3$ since it is triangulated) in Section 2.6. Thus we obtain:

Theorem 2.9.1. The lattice packings $D_{d}$ and $A_{d}$ for $d \geq 3$ are uniformally stable and periodically stable for sublattices.

Remark 2.9.1. One can show that the densest lattice packings (known up to dimension 8) $A_{2}, A_{3}, D_{4}, D_{5}, E_{6}, E_{7}$, and $E_{8}$ are all uniformly stable and periodically stable for sublattices.

\section{Cubic Lattice Packings of Spheres in $\boldsymbol{E}^{d}$ and Uniform Stability}

Theorem 3.1. The densest cubic lattice packing of $E^{d}, d \geq 2$, with unit balls is not uniformly stable.

Proof. We explain our construction in the planar case and note that exactly the same can be done if $d \geq 2$.

Let $\mathcal{P}$ be the densest square lattice packing with unit circles. Given $\varepsilon>0$ we are going to select a subset of $n$ circles of $\mathcal{P}$ (the smaller $\varepsilon$ is, the larger the number $n$ will be) and show that these circles can be rearranged such that each circle is moved by a distance $<\varepsilon$ and the rearranged circles together with the rest of the circles form a packing different from $\mathcal{P}$. 


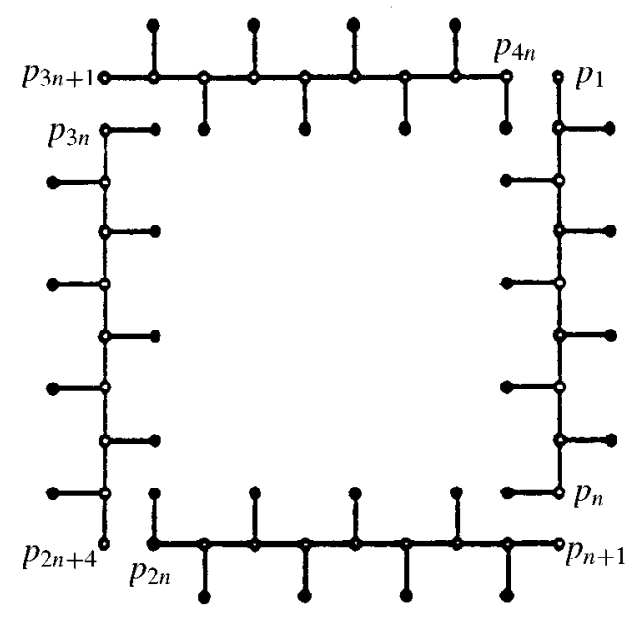

Fig. 5

To ease the description of our argument, first join the centers of the contiguous circles by segments. What we get is a square tiling, where each square has sidelength 2 . On the edge graph of this tile choose a square $\mathrm{Sqr}_{2 n}$ of sidelength $2 n, n$ odd (for a given $\varepsilon$ a sufficiently large odd $n$ will be specified later), and label those vertices of the square tile which lie on the sides of the square $\operatorname{Sqr}_{2 n}$ by $p_{1}, p_{2}, \ldots, p_{4 n}$ in a clockwise order so that $p_{1}$ is-say-the right upper vertex of the square $\operatorname{Sqr}_{2 n}$. Then delete all but a finite number of edges and vertices to get the framework $G_{n}(p)$ shown in Fig. 5. For completeness, here is the precise list of the edges and vertices to be deleted:

(i) delete all edges of the tiling which are not incident with the vertex $p_{i}, i=$ $1, \ldots, 4 n$,

(ii) delete all edges which connect the vertices $p_{2 i+j n}, i=1, \ldots,(n-1) / 2 ; j=$ $0,1,2,3$, to vertices inside the square $\mathrm{Sqr}_{2 n}$,

(iii) delete all edges which connect the vertices $p_{2 i-1+j n}, i=1, \ldots,(n+1) / 2$; $j=0,1,2,3$, to vertices outside the square $\operatorname{Sqr}_{2 n}$,

(iv) delete the edges $p_{n} p_{n+1}, p_{2 n} p_{2 n+1}, p_{3 n} p_{3 n+1}$, and $p_{4 n} p_{1}$,

(v) delete all vertices of the tiling which become disconnected from the vertices $p_{1}, \ldots, p_{4 n}$.

The remainder of the tiling is what we call framework $G_{n}(p)$, assuming that all the edges are bars and the vertices $p_{1}, \ldots, p_{4 n}$ are variable vertices while the rest of the vertices are fixed vertices (Fig. 5). It is clear that this framework has four congruent connected components and that each can be moved independently from the others. We call vertex $p_{1}$ the first and $p_{n}$ the last vertex of the first component. Similarly in clockwise order $p_{n+1}, p_{2 n+1}$, and $p_{3 n+1}$ are the first vertices and $p_{2 n}, p_{3 n}$, and $p_{4 n}$ are the last vertices of the second, third and fourth component. The integer $n$ also measures the lengths of the components. The motions of the first vertices of the components uniquely determine the 
motions of the components. In fact, we show that

Lemma 3.1. For any $\varepsilon>\delta>0$ there is an $n_{0}$ such that if the first vertex $p_{1}$ of the first component of the framework $G_{n}(p), n>n_{0}$, is moved inside the square $\operatorname{Sqr}_{2 n}$ with a distance $<\varepsilon$ such that its distance from $p_{4 n}$ is just greater than 2 , then the last vertex $p_{n}$ of the first component moves with a distance $<\delta$.

Proof. Denote by $p_{i}^{*}$ the position of the vertex $p_{i}$ for each $i=1,2, \ldots, n$ when vertex $p_{1}$ is moved by a distance $<\varepsilon$. Let $h_{i}$ be the length of the perpendicular projection of the segment $p_{i}^{*} p_{i+1}^{*}$ onto the line $p_{i} p_{i+1}$. It is easy to see that the projection of $p_{i}^{*}$ onto the line $p_{i} p_{i+1}$ lies between the vertices $p_{i}$ and $p_{i+1}$ and that $h_{i}$ increases as $i$ increases. Lemma 3.1 claims that, if $n$ is large enough, then the last vertex $p_{n}^{*}$ of the first component remains as close to its original position as we wish. Notice that because $p *_{i}$ is moving on a circle (of radius 2) tangent to the line $p_{i} p_{i+1}$ at $p_{i}$ it is the same as claiming that the limit of the lengths $h_{i}$ as $i$ approaches infinity is 2 . Finally, assume that the limit of the lengths $h_{i}$, as $i$ approaches infinity, is less than 2. Then for large $i$ the projection of $p_{i}^{*}$ onto the line $p_{i} p_{i+1}$ would not lie between the vertices $p_{i}$ and $p_{i+1}$, a contradiction.

Let us move the first vertex of the first component inside the square $\operatorname{Sqr}_{2 n}$ with a distance less than $\varepsilon$ such that its distance from $p_{4 n}$ is just greater than 2 , let us say it is $2+\delta, \delta>0$ (Fig. 6(a)). By Lemma 3.1, if $n$ is a large enough, then the last vertex $p_{n}^{*}$ of the first component remains as close to its original position as we wish. This means that if $n$ is a large enough odd integer, we can find a position for the first vertex of the second component within the $\varepsilon$ neighborhood of $p_{n+1}$ inside the square $\mathrm{Sqr}_{2 n}$, such that its distance from the last vertex of the first component is equal to 2 . The existence of such a position $p_{n+1}^{*}$ inside the square $\operatorname{Sqr}_{2 n}$ is what makes this construction work (see Fig. 6(b)). Notice for contrast that with continuous motion the vertex $p_{n+1}^{*}$ cannot be kept inside the square $\mathrm{Sqr}_{2 n}$. By the same reasoning, if $n$ is large enough, then we can find a position $p_{2 n+1}^{*}$ for the first vertex of the third component within the square $\mathrm{Sqr}_{2 n}$, so that it is at a distance 2 from the last vertex of the second component; and then again, if $n$ is large enough, then we can find a position $p_{3 n+1}^{*}$ for the first vertex of the fourth component within the square $\mathrm{Sqr}_{2 n}$, so that it is at a distance 2 from the last vertex of the third component and still the last vertex of the fourth component is moved by a distance $<\delta$. This means that it lies at a distance $>2$ from the first vertex of the first component, and thus the unit circles placed at the centers $p_{1}^{*}, p_{2}^{*}, \ldots, p_{4 n}^{*}$ neither overlap each other nor the circles placed at the deleted vertices, which completes the proof of Theorem 3.1.

In Section 4 we discuss a general method which might help to determine finite stability of certain packings of unit spheres. Among others it turns out that the densest cubic lattice packing of $E^{d}$ with unit spheres is finitely stable.

\section{Finite Stability of Sphere Packings in $\boldsymbol{E}^{d}$}

Let us start with the following alternate definition (iii*) in Section 1 of finite stability of sphere packings of $E^{d}$ with unit balls. 


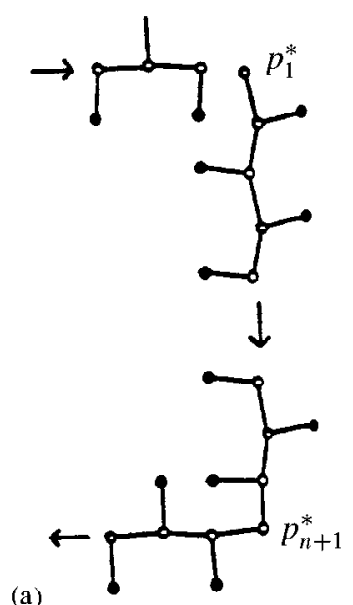

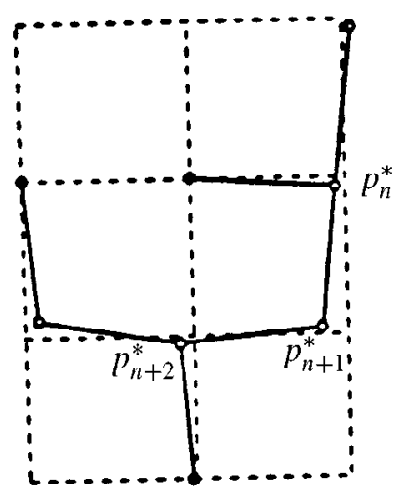

(b)

Fig. 6

(iii*) A sphere packing $\mathcal{P}$ of $E^{d}(d>1)$ with unit balls is finitely stable if and only if for any finite subset $\mathcal{P}^{\prime}$ of the balls of $\mathcal{P}$, there exists an $\varepsilon_{\mathcal{P}^{\prime}}>0$ such that one cannot rearrange the balls of $\mathcal{P}^{\prime}$ such that each ball is moved by a distance less than $\varepsilon_{\mathcal{P}^{\prime}}$ and the rearranged balls together with the rest of the packing form a packing different from $\mathcal{P}$.

Let $\mathcal{P}$ be a packing of $E^{d}(d>1)$ with unit balls. Though the following equivalent condition for finite stability of sphere packings can be proved in a very trivial way it has several applications.

Lemma 4.1. A packing $\mathcal{P}$ of $E^{d}(d>1)$ with unit balls is finitely stable if and only if the strut-framework generated by the graph of the packing $\mathcal{P}$ is finitely rigid.

Corollary 4.1. The densest cubic lattice packing of $E^{d}$ with unit balls is finitely stable.

Proof. Let us recall the following result proved in [C1]: Let $G(p)$ be a tensegrity framework with each member a strut. Then $G(p)$ is rigid if and only if $G(p)$ is infinitesimally rigid. Moreover, we know from [RW] that a tensegrity framework $G(p)$ is infinitesimally rigid if and only if (i) $G(p)$ has a proper equilibrium stress $\omega$, such that $\omega_{i j}$ is nonzero for every $\{i, j\}$ a strut or cable and (ii) the bar framework obtained from $G(p)$ by replacing each member of $G(p)$ with a bar is infinitesimally rigid. Thus, in order to obtain Corollary 4.1 from Lemma 4.1 it is sufficient to prove that the strut-framework generated by the densest cubic lattice packing of $E^{d}$ with unit balls has a negative selfstress. Moreover, the bar framework obtained from the one generated by the packing by replacing each strut with a bar is infinitesimally rigid. As both can be seen easily, we omit details. 


\section{References}

[AR] L. Asimow and B. Roth. The rigidity of graphs II. J. Math. Appl. 68 (1979), 171-190.

[BG] T. Banchoff and P. Giblin. On the geometry of piecewise circular curves. Amer. Math. Monthly 101 (1994), no. 5, 403-416.

[BD] I. Bárány and N. P. Dolbilin. A stability property of the densest circle packing. Monatsh. Math. 106 (1988), no. 2, 107-114.

[B] A. Bezdek. Solid packing of circles in the hyperbolic plane. Studia Sci. Math. Hungar. 14 (1979), 203-207.

[BBC] A. Bezdek, K. Bezdek, and R. Connelly. Finite and uniform stability of sphere coverings. L. Fejes Tóth Festschrift, Discrete Comput. Geom. 13 (1995), nos. 3-4, 313-319.

[C1] R. Connelly. Rigid circle and sphere packings; Part I: Finite packings. Structural Topology 14 (1993), 43-60.

[C2] R. Connelly. The rigidity of certain cabled frameworks and the second-order rigidity of arbitrarily triangulated convex surfaces. Adv. in Math. 37 (1980), no. 3, 272-299.

[CW] R. Connelly and W. Whiteley. Second-order rigidity and pre-stress stability for tensegrity frameworks. SIAM J. Discrete Math. 1-40 (to appear).

[CS1] J. H. Conway and N. J. A. Sloane. Sphere Packings, Lattices and Groups. Springer-Verlag, New York, 1988.

[CS2] J. H. Conway and N. J. A. Sloane. The cell structures of certain lattices. Miscellanea Mathematica (P. Hilton, F. Hirzebruch, and R. Remmert, eds.). Springer-Verlag, New York, 1991, pp. 71-107.

[D] M. Dehn. Über der Starrheit Konvexer Polyeder. Math. Ann. 77 (1916), 466-473.

[F1] L. Fejes Tóth. Regular Figures. Pergamon Press, Oxford, 1964.

[F2] L. Fejes Tóth, Lagerungen in der Ebene, auf der Kugel und im Raum (2. Aufl.). Springer-Verlag, New York, 1972.

[G] C. F. Gauss. Untersuchungen über die Eigenschaften der positiven ternären quadratischen Formen von Ludwig August Seber, Göttingische gelehrte Anzeigen, 1831 Juli 9. J. Reine Angew. Math. 20 (1840), 312-320.

[Gl] H. Gluck. Almost all simply connected closed surfaces are rigid. In: Geometric Topology. Lecture Notes in Mathematics, vol. 438. Springer-Verlag, Berlin, 1975, pp. 225-239.

[H] J. F. P. Hudson. Piecewise Linear Topology. W. A. Benjamin, New York, 1969.

[R] O. Reynolds. On the dilatancy of media composed of rigid particles in contact. Phil. Mag. and J. Sci. 5 (1885), 469-481.

[RS] C. P. Rourke and B. J. Sanderson. Introduction to Piecewise-Linear Topology. Springer-Verlag, New York, 1972.

[RW] B. Roth and W. Whiteley. Tensegrity frameworks. Trans. Amer. Math. Soc. (1981), 410-446.

[S] M. Spivak. Differential Geometry, Vol. 1, Chapter 7. 1970.

[Wa] A. Wallace. Algebraic approximation of curves. Canad. J. Math. 10 (1958), 248-278.

[W] W. Whiteley. Infinitesimally rigid polyhedra I: statics of frameworks. Trans. Amer. Math. Soc. 285 (1984), 431-465.

Received April 22, 1996, and in revised form October 11, 1996. 